\title{
New Electrostatic Phase Plate for Transmission Electron Microscopy and its Application for Wave-Function Reconstruction
}

\author{
K. Schultheiss, ${ }^{*}$ J. Zach, ${ }^{* *}$ B. Gamm, ${ }^{*}$ M. Dries, ${ }^{*}$ N. Frindt,*** R.R. Schröder*** and D. \\ Gerthsen* \\ * Laboratorium für Elektronenmikroskopie, Karlsruher Institut für Technologie, D-76128 Karlsruhe, \\ Germany \\ ** CEOS GmbH, Englerstr. 28, D-69126 Heidelberg, Germany \\ *** BioQuant CellNetworks, Universität Heidelberg, D-69120 Heidelberg, Germany
}

Achieving phase contrast in transmission electron microscopy (TEM) for weak-phase objects by physical phase plates has recently been an intensively studied topic. Phase plates positioned in the back focal plane (BFP) of the objective lens are used to achieve a relative phase shift of the scattered electrons with respect to the transmitted beam. Two main concepts exist: thin-film phase plates [1,2] and electrostatic phase plates like the Boersch-phase plate [3] with the possibility to adjust the phase shift by varying the applied voltage.

Boersch phase plates consist of a ring-shaped micro lens, which is positioned around the transmitted beam in the BFP and hence blocks electrons of a complete spatial frequency band. Even though the device dimensions have been further reduced the effect of the ring structure still dominates the images. We present a promising novel type of an electrostatic phase plate consisting of a microcoaxial cable analogue (FIG. 1 a \& b), which is placed in the vicinity of the transmitted beam [4]. When applying a voltage a well localized electrostatic potential builds up at the phase-plate tip (FIG. $1 \mathrm{c})$. The new phase plate overcomes the drawback of a frequency-blocking ring electrode.

Experimental phase-contrast images of PbSe nanoparticles are presented (FIG. 2) which are compared to conventional images at different defocus values (FIG. 2 a-c). The phase contrast clearly varies and depends on the applied voltage and the consequently resulting phase shift of the unscattered electrons (FIG. 2 d-f). We also show that phase contrast is not significantly impaired by the inhomogeneous electrostatic potential.

We present for the first time the reconstruction of an object wave function using an electrostatic phase plate with platinum particles as test objects. The new reconstruction method is based on a series of only three experimental phase-contrast TEM images. The reconstruction technique was suggested recently by our group [5] and is not limited to weak-phase objects and linear image formation.

References

[1] R. Danev et al., Ultramicroscopy 88 (2001) 243.

[2] R. Danev et al., J. Phys. Soc. Jpn. 73 (2004) 2718.

[3] K. Schultheiß et al., Rev. Sci. Instrum. 77 (2006) 033701.

[4] J. Zach, patent pending: WO/2008/061603, PCT/EP2007/009289.

[5] B. Gamm et al., Ultramicroscopy, DOI: 10.1016/j.ultramic.2010.02.006. 
[6] The project is funded by the German Research Foundation (Deutsche Forschungsgemeinschaft) under Ge 841/16 and Sch 424/11.
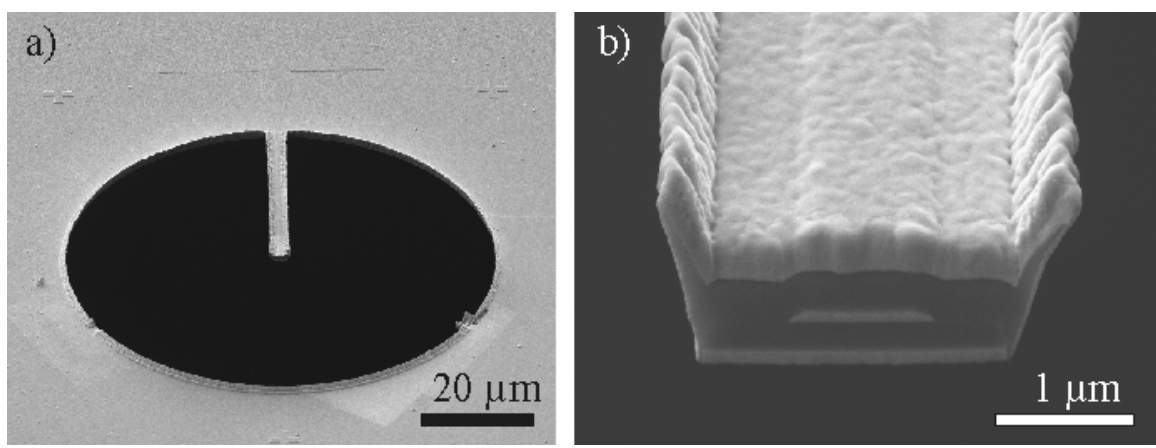

c)

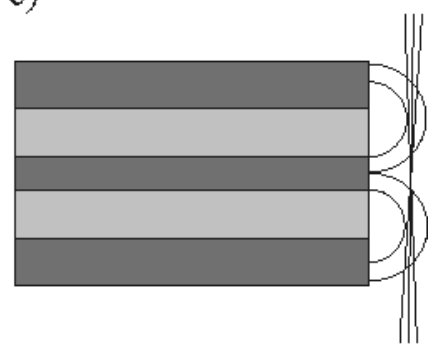

FIG. 1. a) Prototype of the new electrostatic phase plate. Scanning electron microscopy image (SEM) of the whole phase plate. b) Magnified SEM image of the phase plate tip. c) Schematic crosssection of the phase plate with an electrostatic field at the tip where the unscattered electrons pass.
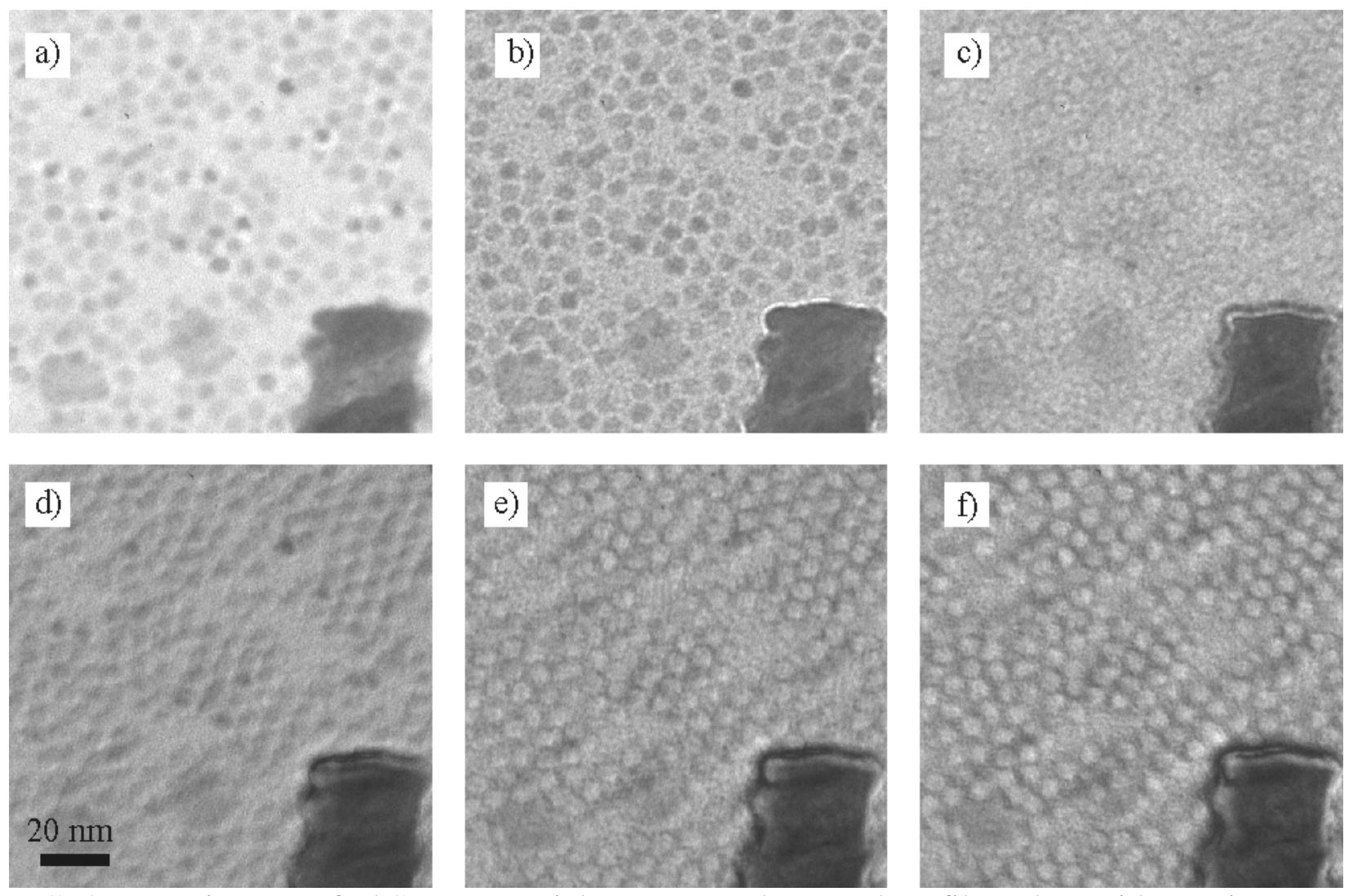

FIG. 2. TEM images of PbSe nanoparticles on amorphous carbon film taken with a Zeiss EFTEM 923 FEG. a-c) Conventional TEM images without phase plate and varying defocus values $\mathrm{Z}$. a) Gaussian focus b) $Z=-970 \mathrm{~nm}$ and c) $\mathrm{Z}=1980 \mathrm{~nm}$. d-f) Phase-plate TEM images with different applied phase shifts $\varphi_{\mathrm{PP}}:$ d) $\varphi_{\mathrm{PP}}=0$, e) $\varphi_{\mathrm{PP}}=\pi / 4$ and f) $\varphi_{\mathrm{PP}}=\pi / 2$ at the same defocus value of 730 $\mathrm{nm}$. 